\title{
Fractal Geometry-Based Hypergeometric Time Series Solution to the Hereditary Thermal Creep Model for the Contact of Rough Surfaces Using the Kelvin-Voigt Medium
}

\author{
Osama M. Abuzeid, ${ }^{1}$ Anas N. Al-Rabadi, ${ }^{2}$ \\ and Hashem S. Alkhaldi ${ }^{1}$ \\ ${ }^{1}$ Mechanical Engineering Department, The University of Jordan, Amman 11942, Jordan \\ ${ }^{2}$ Computer Engineering Department, The University of Jordan, Amman 11942, Jordan
}

Correspondence should be addressed to Anas N. Al-Rabadi, a.alrabadi@ju.edu.jo

Received 28 January 2010; Accepted 23 May 2010

Academic Editor: Ming Li

Copyright (C) 2010 Osama M. Abuzeid et al. This is an open access article distributed under the Creative Commons Attribution License, which permits unrestricted use, distribution, and reproduction in any medium, provided the original work is properly cited.

This paper aims at constructing a continuous hereditary creep model for the thermoviscoelastic contact of a rough punch and a smooth surface of a rigid half-space. The used model considers the rough surface as a function of the applied load and temperatures. The material of the rough punch surface is assumed to behave as Kelvin-Voigt viscoelastic material. Such a model uses elastic springs and viscous dashpots in parallel. The fractal-based punch surface is modelled using a deterministic Cantor structure. An asymptotic power law, deduced using approximate iterative relations, is used to express the punch surface creep which is a time-dependent inelastic deformation. The suggested law utilized the hypergeometric time series to relate the variables of creep as a function of remote forces, body temperatures, and time. The model is valid when the approach of punch surface and half space is in the order of the size of the surface roughness. The closed-form results are obtained for selected values of the system parameters; the fractal surface roughness and various material properties. The obtained results show good agreement with published experimental results, and the methodology can be further extended to other structures such as the Kelvin-Voigt medium within electronic circuits and systems.

\section{Introduction}

Surface topography plays a significant role in tribology, that is, in problems of friction, wear, lubrication, and contact [1]. Therefore, the problem of analysis of rough surfaces attracts the attention of engineers and applied mathematicians. Historically, the following engineering parameters, statistical in nature, were used for the characterization of surface roughness: (1) the root mean square of the heights, $\sigma,(2)$ the root mean square of slopes, $\sigma_{m}^{2},(3)$ and the root 
mean square of curvatures, $\sigma_{k}^{2}$. However, it was realized that the topography of engineered surfaces is too complex to be described completely by a few statistical parameters. Thus, it was found that roughness has a multiscale nature and requires sophisticated mathematical techniques for its description.

First attempts to model the distribution of heights of surface asperities utilize the classical random field theory which assumed that the functions of surface model are differentiable. In particular, this implies that limiting values for $\sigma_{m}^{2}$ and $\sigma_{k}^{2}$ should exist as the sample interval tends to " 0 " [1]. However, it turned out that such limiting behavior is in contradiction with the results of advanced investigations of surfaces. For example, the exponential behavior of the autocorrelation function implies that the engineering parameters should tend to infinity rather than to constant values when the sampling interval is infinitely reduced [2]. Furthermore, it was shown that the profiles of a large number of both natural and artificial surfaces have the following form of the spectral density function $G(\omega) \sim 1 / \omega^{v}$ where $v \approx 2$, and $\omega$ is the spatial frequency (see (1.13)). It follows from this that all wavelengths are equally represented in the profile and that there exists no characteristic scale; in other words, after arbitrary magnification roughness looks like before. Moreover, it was found that the values of engineering parameters depend on the measurement scale, that is, these parameters are scale dependent $[2,3]$.

The fractal approach was introduced as an attempt to give a scale-invariant characterization of surface topography. The idea of fractality of roughness was experimentally verified on real surfaces as well as when applied to mathematically simulated profiles [4]. Figure 1 shows a picture of popular fractals, that is, the middle-third Cantor set, the von Koch curve, graph of the Weierstrass-Mandelbrot function $C$ in the range $0 \leq x \leq 3(p=1.5$ and $\gamma=0.5$ where $p$ and $\gamma$ are two numerical parameters (see (1.17)) where the trend of the function is $\sim x^{2}$, and trajectories of a fractional Brownian process for different Hurst index $H$ and fractal dimension $D$ [5-7]. The index $H$ is the key parameter of the fractal surface which describes the smoothness of the surface.

Evidently, roughness of the surface of a body has a great influence on stress fields that arise when two deformable bodies are pressed together. Analysis of the effect of roughness on the contact interaction of solids has attracted wide attention [8].

One of the most popular models for studying contact of rough bodies is the Greenwood and Williamson (GW) model based on the use of the Hertz theory [9], where it is important to mention that (GW) model is a nonscale-invariant [10]. Currently, the development of models of contact between nominally flat fractal rough surfaces presented for the Cantor profile is an active area of research [11]. Various contact problems utilizing Cantor profile were considered [12-17]. All these models consider the one-level Cantor profile. It is argued that such profile is simple for analytical analysis. However, it has a minor drawback: all asperities of the profile have one-level character, while all real roughness has a hierarchical structure [17].

It is accepted that fractal dimension is not a compressive geometric parameter that could characterize alone the behavior of contacting rough bodies [1]. Moreover, the employment of the fractal approach in the study of surfaces has several drawbacks. The proposed model can be both fractal and nonfractal depending on values of the structural parameters. Regardless of this, the model profile remains rough and possesses certain selfaffine properties. The iterative regular construction of the profile allows us to analyze its prestructures, that is, prefractals, of arbitrary generation.

In this introduction, important and relevant definitions and methods that are attributed to fractal geometry with the application to the modeling of rough surfaces will be 


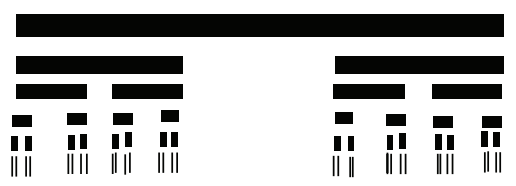

(a)

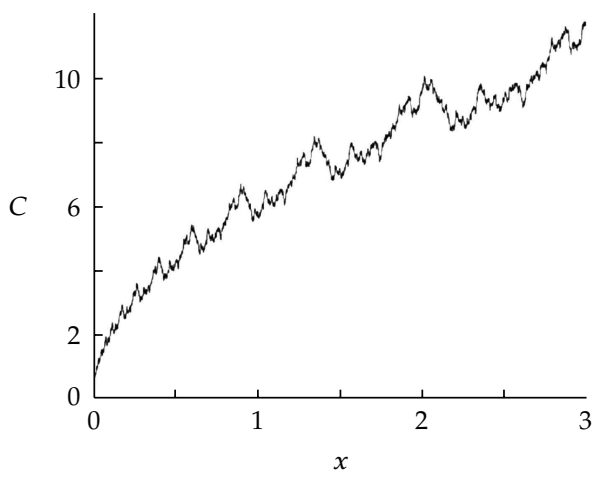

(c)

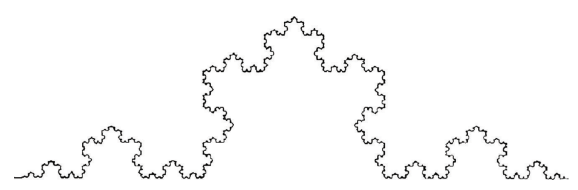

(b)

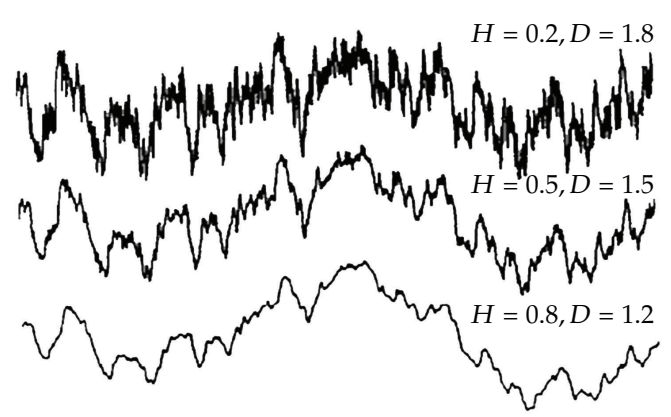

(d)

Figure 1: Common fractals: (a) the middle-third Cantor set, (b) the von Koch curve, (c) the WeierstrassMandelbrot function $C$ in the range $0 \leq x \leq 3(p=1.5$ and $\gamma=0.5)$, and (d) trajectories of a fractional Brownian process for different $H$ and $D$.

fully presented. Furthermore, the important differences between mathematical and utilized physical fractals will be explicitly highlighted.

\subsection{Mathematical Definition of Fractals}

Mandelbrot stated that a set in a metric space is called a fractal set if the Hausdorff-Besicovitch dimension of the set is greater than its topological dimension [18]. Let $X$ be a compact metric space and $O$ be the totality of open balls in X. The Hausdorff $s$-measure of a subset $S \subset X$ which is defined for $s \geq 0$ as the following limit:

$$
m_{H}(S, s)=\lim _{\sigma \rightarrow 0} \inf _{G \in O}\left\{\sum_{V \in G}(\operatorname{dim} V)^{s}: S \subseteq \bigcup_{V \in G} V, \operatorname{diam} V \leq \delta\right\} .
$$

Here $G$ is finite or denumerable subset of $O$. It was proven that there exists a value $s_{0}$ such that

$$
m_{H}(S, s)= \begin{cases}\infty, & \text { for } s<s_{0} \\ 0, & \text { for } s>s_{0}\end{cases}
$$

The Hausdorff dimension of the set $S$, denoted by $\operatorname{dim}_{H} S$, is the number $S_{0}$ such that (1.2) holds. Unfortunately, the calculation of the Hausdorff dimension of mathematical objects often demands a lot of effort. Even to find some estimations of the dimension, it is necessary to overcome a number of rather complex mathematical difficulties [19]. This issue 
called for the use of other definitions of dimension which are useful in applied mathematics for the characterization of fractal objects. One such alternative is the box dimension [1]. The analytical calculation of the box dimension is usually easier since the corresponding definition of this dimension involves coverings by spheres of equal radii.

Let $E$ be the Euclidean dimension of the space in which a set $S$ is embedded. For $\delta>0$, let $N(\delta)$ be the smallest number of $E$-dimensional balls or cubes of diameter $d$ needed to cover the set $S$. The box counting dimension or box dimension, denoted by $\operatorname{dim}_{B} S$, can be defined if the following limit exists:

$$
\operatorname{dim}_{B} S=\lim _{\delta \rightarrow 0+} \frac{\log N(\delta)}{-\log \delta}
$$

It can be proven that $\operatorname{dim}_{B} S$ does not change if one takes $N(\delta)$ to be either the smallest number of $\delta$-cubes that cover $S$ : the number of $\delta$-mesh cubes that intersect $S$; or the smallest number of sets of diameter at most $d$ that cover $S$; or the largest number of disjoint $\delta$-balls with centers in $S$. Unfortunately, the box dimension is not always equal to the Hausdorff dimension. For example, the set $S=\{0,1,1 / 2, \ldots, 1 / n \ldots\}$ has unequal values for the Hausdorff and box dimensions for $\operatorname{dim}_{H} S \neq \operatorname{dim}_{B} S=1 / 2$. However, it can be proven that $\operatorname{dim}_{H} S \leq \operatorname{dim}_{B} S$.

As a simple alternative to the Hausdorff measure, we can introduce the $s$-measure $m_{s}$ of a set as the following limit:

$$
m_{S}(S)=\lim _{\delta \rightarrow 0+} N(\delta) \delta^{S}
$$

and define the box dimension as the value $s=D$ such that $m_{S}(S)$ has a jump from 0 to $\infty$ similar to the behavior of $m_{H}(S, s)$ in (1.2).

On the other hand, the difficulties involved with calculating the Hausdorff dimension are the reason for the opinion that the Hausdorff dimension is not generally used in applications in the study of fractal and non fractal curves that are originated in other sciences such as in biology, engineering, physics, quantum physics and computing [20-27].

\subsection{Physical Concept of Fractals}

Evidently, it is impossible to carry out the scaling procedure for any real physical object down to infinitely small scales. Hence, the mathematical concept of the Hausdorff measure is applicable only to mathematical models of objects rather than to the objects themselves and, of course, the Hausdorff dimension cannot be obtained by experimental procedures. In this sense there are no actual fractal objects in nature. For physical objects, the box dimension cannot be calculated analytically but it is estimated by experimental or numerical calculations. However, various errors can arise during such numerical calculations. There is no canonical definition of physical fractals and there are numerous methods for the practical estimation of the fractal dimension of an object. The cluster fractal dimension is taken as the first example of a physical fractal dimension definition.

Let a whole cluster be imagined as consisting of elementary parts of the size $\delta_{*}[1]$. An object can be modeled as a fractal cluster with dimension $D$ when the model considers scales $R$ such that $\delta_{*}<R<\Delta_{*}$, where $\delta_{*}$ and $\Delta_{*}$ are the upper and lower cutoffs for the fractal 
representation. To get the value $D$ of the dimension, the considered region is discretized into cubes with side length $\delta_{*}$. Then the smallest number of $E$-dimensional cubes needed to cover the cluster $\left(N\left(\delta_{*}\right)\right)$ is counted. One says that the cluster is fractal if the numbers $N\left(\delta_{*}\right)$ satisfy the so-called number-radius relation for different sizes of the considered region of the cluster $R$ as follows:

$$
N\left(\delta_{*}\right) \approx\left(\frac{R}{\delta_{*}}\right)^{D}, \quad \delta_{*}<R<\Delta_{*}
$$

The value of $D$ is estimated as the slope of linear growth of $\ln \left(N\left(\delta_{*}\right)\right)$ plotted against $\ln (R)$. The power $D$ is usually called the cluster dimension or mass dimension.

In literature, various methods were utilized to estimate the fractal dimension of a physical object. However, the notion of fractal dimension is not well-defined in that the relative value does depend on the approach used. Indeed, only for the mathematical box dimension of a fractal set $S$ it is proven that $\operatorname{dim}_{B} S$ is the same when using various specific schemes of covering [28], while for physical fractals the estimations of the fractal dimension inevitably involve various techniques, distinct scale ranges, and various computation rules. Therefore, the obtained values can differ strongly and it is unlikely that they could be fruitfully compared for distinct objects. Thus, even in the case of physical objects of a similar nature, it would be wrong to consider the fractal dimension of these objects as their specific property without referring to the estimation technique involved.

\subsection{Self-Similarity and Self-Affinity of Surfaces}

Let us recall that a one-to-one mapping $M$ of a plane $\pi$ onto a plane $\pi^{\prime}$ is called a similarity mapping with coefficient $\lambda>0$, or simply a similarity, when the following property holds: if $\{A, B\}$ are any two points of $\pi$, and $\left\{A^{\prime}, B^{\prime}\right\}$ are their images under $M$, then $\left|A^{\prime} B^{\prime}\right|=\lambda|A B|$ [29]. It is known that any similarity transformation of a plane is a homogeneous (isotropic) dilation of coordinates $\left\{x^{\prime}=\lambda x, z^{\prime}=\lambda z\right\}$ up to a rotation and translation. A set $S$ is called statistically self-similar if under homogeneous scaling with the coefficient $\lambda$, where $1>\lambda>0$, it is identical from the statistical point of view to the set $S^{\prime}=\lambda S$.

In practice, it is impossible to verify that all statistical moments of the two distributions are identical. Frequently, a set $S$ is said to be self-similar if only a few moments do not change under scaling [30]. A one-to-one mapping $M$ of a plane $\pi$ onto a plane $\pi^{\prime}$ is called an affine mapping, if the images of any three collinear points are collinear in turn [29]. In general, an affine transformation of a plane may be given in any coordinate system as a nondegenerative linear transformation. In practical studies of rough surfaces, one often considers a particular affine mapping, with anisotropic scaling, that is given coordinate wise by $x^{\prime}=\lambda x$ and $z^{\prime}=$ $\lambda^{H} z$. Here $z$ is a graph of a surface profile and $H$ is some scaling exponent.

One says that a fractal is self-affine if it is invariant from the statistical point of view under quasihomogeneous (anisotropic) scaling. It is possible to show that usually a quasihomogeneous transformation is a particular case of Lipschitz homeomorphism [1,17]. The Hausdorff dimension of a set $S$ does not change under the action of the Lipschitz homeomorphism $L$ as follows:

$$
\operatorname{dim}_{H} S=\operatorname{dim}_{H} L(S)
$$


The ideas of self-similarity and self-affinity are very popular in studying surface roughness because experimental investigations show that usually profiles of vertical sections of real surfaces are statistically similar to themselves under repeatedly magnifications; however, the profiles should be scaled differently in the direction of nominal surface plane and in the vertical direction. The self-affine fractals were used in a number of papers as a tool for description of rough surfaces $[3,31,32]$. Two standard examples of self-affine fractals are the trace of the fractional Brownian motion and the Weierstrass function. The former is a statistical fractal while the latter is a deterministic fractal.

\subsection{Brownian Surfaces and Random Fractals}

Fractional Brownian processes are widely used in creating computer-generated surfaces, in particular landscapes. For example, a profile can be constructed as a graph of $1 D(f B m) V_{H}(x)$ of index $H$, where $x$ is taken as the time and $z$ is the random variable of the single valued function $V_{H}(x)$ with the following property:

$$
\left\langle\left[V_{H}(x+\delta)-V_{H}(x)\right]^{2}\right\rangle \sim \delta^{2 H}, \quad 0<H<1
$$

where \langle\rangle denotes averaging over the ensemble, and $H$ is the Hurst index. The scaling behavior of the different traces, $V_{H}(x)$, is characterized by a particular $H$ which relate the typical change in $\Delta z(x)$, where $z(x)=V_{H}(x)$, is the trace of the $f B m$, and the change in the spatial coordinate $\Delta x$ by the simple scaling law $[30,33,34]$ :

$$
\Delta z(x) \sim \Delta x^{H}
$$

It is known that, with probability equal to " 1 ", the following holds [28]:

$$
\operatorname{dim}_{H} V_{H}(x)=\operatorname{dim}_{B} V_{H}(x)=2-H .
$$

The autocorrelation function is one of the main tools for studying statistical models of rough surfaces. The autocorrelation function $R(\delta)$ of the profile is

$$
R(\delta)=\lim _{T \rightarrow \infty} \frac{1}{2 T} \int_{-T}^{T}[z(x+\delta)-\bar{z}][z(x)-\bar{z}] d x=\langle[z(x+\delta)-\bar{z}][z(x)-\bar{z}]\rangle
$$

or

$$
R(\delta)=\lim _{T \rightarrow \infty} \frac{1}{2 T} \int_{-T}^{T} z(x+\delta) z(x) d x-(\bar{z})^{2}
$$

where $\bar{z}$ is the average value of the profile function $z(x)$. 
Another tool for the characterization of surfaces is the spectral density function $G(\omega)$ which is the Fourier transform of $R(\delta)$ :

$$
\begin{aligned}
& G(\omega)=\frac{2}{\pi} \int_{0}^{\infty} R(\delta) \cos \omega \delta d \delta \\
& R(\delta)=\frac{2}{\pi} \int_{0}^{\infty} G(\omega) \cos \omega \delta d \omega .
\end{aligned}
$$

In general, it is accepted in fractional Brownian motion that [14]:

(i) if the autocorrelation function $R(\delta)$ of the profile $z(x)$ satisfies $R(0)-R(\delta) \sim \delta^{2(2-s)}$, then it is reasonable to expect that the box dimension of the graph $z(x)$ is equal to $s$, note that one can find $R(0)-R(\delta) \sim \delta^{2 H}$ for the $f B m$ defined by (1.7).

(ii) if the profile $z(x)$ has spectral density:

$$
G(\omega) \sim \frac{1}{\omega^{v}}
$$

then it is reasonable to expect that the box dimension of the graph $z(x)$ is equal to $(5-v) / 2$ [1]. The above conclusions are valid for mathematical models of the profile, for which the relation $2(5-s)=v-1$ or $v=5-2 s$ holds. The exponent $v$ varies typically between 0 and 2 . Usually, it is assumed that the same conclusions concerning the box dimension are valid for physical fractals as well. It is shown that real surfaces approximately satisfy the property in (1.13) in wide range of scales [35]. The moments $m_{n}$ of the spectral density $G(\omega)$ provide a useful description of the surface roughness:

$$
m_{n}=\int_{\omega_{0}}^{\infty} \omega^{n} G(\omega) d \omega
$$

where $\omega_{0}=2 \pi / \lambda_{0}$ is the wave number corresponding to the profile length $\lambda_{0}$. It is possible to show that $m_{0}$ is the variance of heights ( $r m s$ height) of the surface, $m_{1}$ is the variance of slopes (rms slope) and $m_{3}$ is the variance of curvatures (rms curvature) [36].

\subsection{Weierstrass-Type Functions and Modeling of Rough Surfaces}

A number of researchers have used the Weierstrass-type functions for fractal modeling of surface roughness [3,31,32] and fractal modeling applications such as in quantum computing $[20,21]$. The real Weierstrass-type function can be defined as:

$$
W(x ; p)=\sum_{n=0}^{\infty} p^{-\gamma n} h\left(p^{n} x\right), \quad p>1,0<\gamma<1
$$


where $h$ is a bounded Hölder function of order greater than $\beta$. The following complex generalization of the $W(x ; p)$ was considered:

$$
\widetilde{W}(x ; p)=\sum_{n=-\infty}^{\infty} p^{-\gamma n}\left[\left(1-e^{i p^{n} t}\right) e^{i \Phi_{n}}\right], \quad p>1,0<\gamma<1,
$$

where $\Phi_{n}$ are arbitrary phases [29].

The Weierstrass-type functions are continuous everywhere and differentiable nowhere. In addition, their graphs are curves whose fractal dimension exceeds one. Fractal properties of these functions including the Weierstrass-Mandelbrot (WM) function $C$ and the Takagi-Hopson function $T$ :

$$
\begin{gathered}
C(x ; p)=\sum_{n=-\infty}^{\infty} p^{-\gamma n}\left(1-\cos p^{n} x\right), \quad p>1,0<\gamma<1, \\
T(x ; p)=\sum_{n=-\infty}^{\infty} p^{-\gamma n}\left|p^{n} x-\left[p^{n} x+\frac{1}{2}\right]\right|, \quad p>1,0<\gamma<1,
\end{gathered}
$$

have been studied in numerous papers $[2,14,29,31]$. By direct calculations, one may obtain:

$$
|\widetilde{W}(x+\delta ; p)-\widetilde{W}(x ; p)| \sim \delta^{r}
$$

which is similar to the behavior of (1.7) of fractional Brownian motion. The box dimension of the Weierstrass function graphs is $D=2-\gamma$ and it is believed that their Hausdorff dimension is the same [28, 37]. Currently, the only known bounds for the Hausdorff dimensions are $D-(c / \log p) \leq \operatorname{dim}_{H}$ graph $C \leq D$, provided that $p$ is large and constant $c$ is large enough [19]. It is possible to calculate the spectral density of the WM function $\widetilde{W}(x ; p)$ as follows:

$$
G(\omega)=\sum_{n=-\infty}^{\infty} \frac{\delta\left(\omega-p^{n}\right)}{p^{2(2-D) n}}
$$

where $\delta$ is the Dirac delta function. Some arguments for approximating this discrete spectral density by a continuous spectral density $\widetilde{G}(\omega) \sim 1 / \omega^{5-2 D}$, whose exponent (5-2D) is in agreement with (1.13) with respect to the box dimension were suggested [2]. The following truncated WM function

$$
\widetilde{W}_{1}(x ; p)=A^{(D-1)} \sum_{n=n_{1}}^{\infty} p^{(D-2) n} \cos 2 \pi p^{n} x
$$

is often used for fractal characterization of the surface topography [3, 31, 32]. Here $n_{1}$ is an integer number, which corresponds to the low cutoff frequency of the profile, and $A$ is the socalled characteristic length scale of the profile. The number $n_{1}$ depends on the length $L$ of the sample and is given by $p^{n_{1}}=1 / L$ and the parameter $A$ determines the position of the spectral density along the $\log G$ axis. It was stated that both parameters $A$ and $D$ of the function $W_{1}$ are 
scale-invariant characteristics of the roughness. However, the extensive experimental studies of this fractal characterization model showed that the values of parameters $A$ and $D$ are not unique and depend on instruments or resolution of a given instrument [1].

Evidently, the function $C(x ; p)$ is not homogeneous. Nevertheless, it exhibits the property $C\left(p^{k} x ; p\right)=p^{k r} C(x ; p)$, with $k \in Z$ where $Z$ is the set of all integers which looks similar to the definition of a homogeneous function $h_{d}$ of degree $d$, that is, $h_{d}(\lambda x)=\lambda^{d} h_{d}(x)$ for $\lambda>0$.

Thus, the graph of the function $C(x ; p)$ near any point $x_{0}$ is repeated in scaling form near all points $p^{k} x_{0}, k \in Z$. This scaling (self-affine) property was often attributed to fractal features of the graph. However, this discrete scaling property is the main property of the socalled parametric-homogeneous $(\mathrm{PH})$ functions introduced $[1,17]$ which strictly satisfy the equation $b_{d}\left(p^{k} x ; p\right)=p^{k d} b_{d}(x ; p)$, with $k \in Z$ where $d$ is degree of homogeneity. As examples of 1-dimensional fractal $\mathrm{PH}$-curves we can consider the graphs of functions $b_{1}$ and $b_{2}$ with degrees $d=1$ and $d=2$, respectively:

$$
\begin{gathered}
b_{0}(x ; p)=x^{-\gamma} C(x ; p), \\
b_{1}(x ; p)=x b_{0}(x ; p) \\
b_{2}(x ; p)=x^{2} b_{0}(x ; p) .
\end{gathered}
$$

Because of (1.6), these functions have the same Hausdorff dimension as the WM function $C(x ; p)$ whose box-dimension is $D$.

Another consequence is that the WM function $C(x ; p)$, with $C(x ; p) \sim x^{2-D}$ can be used only as an example of fractal profile and it cannot be considered as the general fractal functional model for simulations of the rough surface profiles. The assumption that the WM function represents the general fractal properties of rough profiles can lead to wrong conclusions concerning surface roughness parameters and their distributions.

The solution to the problem of mechanical contact between elastically deforming solids was obtained by Hertz [8]. Subsequently, several approaches were used to analyze the contact interaction between the soft layer and the indenting object surface [38-42]. These methods are based upon Radok's technique of replacing the elastic constants in the elastic solution by the corresponding integral or differential operators, which appear in the stress-strain relations for linear viscoelastic materials. Furthermore, these studies assumed that the surfaces of contacting solids are smooth, excluding from consideration all real solids, which have a certain degree of roughness and waviness regardless of how fine their finish is [43].

Various models for the approach of the fractal punches were considered [11-16]. In the previously cited works, different constitutive relations were considered: (1) linear elastic material [11], (2) rigid-perfectly plastic material [13], (3) elastic-perfectly plastic material [12], (4) linear viscoelastic creep model via Maxwell medium [14], (5) linear viscoelastic creep model via standard linear solid (SLS) material [15], and (6) linear thermoviscoelastic relaxation model via Maxwell medium [16].

The objective of this work is to introduce an alternative approach, using fractal geometry, to study the deformation of a viscoelastic surface as a function of the force applied and the bulk temperature. In this model friction force effect is assumed to be negligible. The development of the fractal model of the rough surface is carried out using fractional Brownian motion in conjunction with Cantor set. The Radok's technique [44] is then used to derive the thermoviscoelastic model from the corresponding elastic model. The main contribution of 
this work is a mathematical model for the time-dependent-creep of a rough surface (cf. (6.7)). This model relates the creep to time, temperature, external applied load, fractal dimension of the rough surface, and various material properties.

Section 2 presents the fractal model, where the Cantor structure is built and its fractal dimension is presented. Section 3 presents the discrete and continuous elastic model. In Sections 4 and 5, the effect of temperature on the viscoelastic behavior is presented and the Arrhenius's relation is introduced. In Section 6, the elastic viscoelastic correspondence is presented which consists of replacing the elastic constant in the elastic solution by the corresponding integral or differential operators from the viscoelastic stress-strain relations. Also, in Section 6 a new continuous model for the creep contact of a thermovisco-elastic punch is presented. In Section 7, the results obtained from the new model is presentd and compared with an experimental results obtained from literature. In Section 8, conclusions and future work are presented.

\section{Fractal Model}

The surface profile of the punch, in contact with a rigid half-space, will be constructed on the basis of Cantor set [11]. The contacting surface is constructed by joining the segments obtained at successive stages of the construction of a Cantor set to one another, Figure 2, where $L_{0}$ correspond to the profile nominal length, and $h_{0}$ is equal to the twice $r m s$ height of the roughness.

At each stage of profile construction, the middle section of each initial segment is discarded so that the total length of the remaining segments is $1 / a$ times the length of the initial segment, where $a>1$. The depth of the recesses (measured from the last step) at the $(i+1)$ th construction step of the fractal surface is $1 / b$ times less than the depth of the $i$ th step, where $b>1$. From this it can easily be shown that the horizontal length and recess depth of the $(i+1)$ th step are, respectively

$$
\begin{aligned}
& L_{i+1}=a^{-1} L_{i}=a^{-(i+1)} L_{0}, \\
& h_{i+1}=b^{-1} h_{i}=b^{-(i+1)} h_{0},
\end{aligned}
$$

where it is assumed that the surface is smooth in a direction perpendicular to the plane of the page. This restriction is not expected to have a significant effect since it is possible to construct a fractal Cantor surface perpendicular to the plane of the page [11].

At the $i$ th generation, the Cantor structure contains $N=2^{i}$ segments, each of length $\delta_{i}=(2 a)^{-i} L_{0}[11]$. The profile of the surface in Figure 2 can be considered as a certain graph of a step function.

It can be seen that, during an iterative step in constructing the surface, scaling in the horizontal direction is $\Delta X_{i+1}=(2 a)^{-1} \Delta X_{i}$, while in the vertical direction, the corresponding fluctuations $\Delta z_{i}$ at the $i$ th generation can be defined by considering the probability of obtaining the value, $z_{i}=b^{-i} h_{0}$.

The fluctuation $\Delta z_{i}$ at the $i$ th generation can be obtained by assuming the $\Delta z_{i}$ scales as the expected value $z_{i} P\left(z_{i}\right)$ in which $\Delta z_{i} \propto z_{i} P\left(z_{i}\right)$ [8], where $P\left(z_{i}\right)$ is the probability of obtaining the value $z_{i}$, that is, $P\left(z_{i}\right)=\left(L_{i}-L_{i+1}\right) / L_{0}$, and it is found that $P\left(z_{i}\right)=a^{-i}(1-1 / a)$.

Thus, the expected value of the fluctuation at the $(i+1)$ th generation is related to the expected value of the fluctuation at the $i$ th generation through $z_{i+1} P\left(z_{i+1}\right)=(a b)^{-i} z_{i} P\left(z_{i}\right)$. 


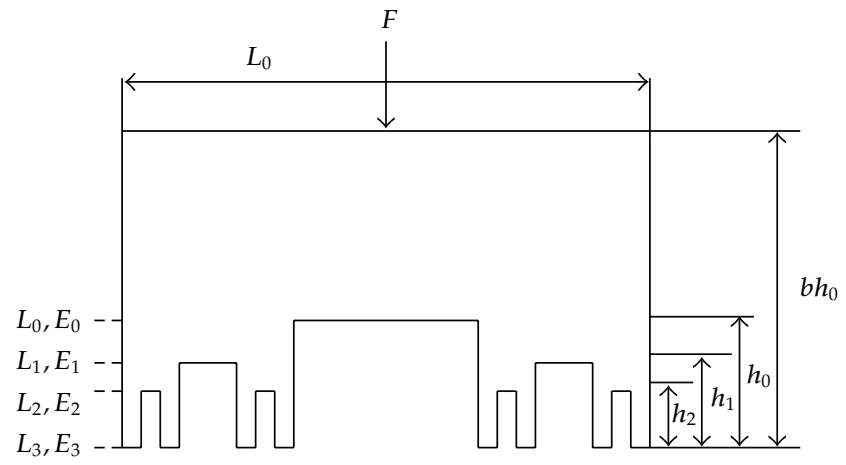

Figure 2: The fractal middle-third Cantor structure, where $E_{0}$ is the initiator step, $E_{1}, E_{2}$, and $E_{3}$ are the other generated step of cantor structure, $L$ 's are the lengths of the $E^{\prime}$ s steps, $h$ 's are the heights of $E^{\prime}$ s steps, and $F$ is the applied load.

Hence $\Delta z_{i+1}=(a b)^{-i} \Delta z_{i}$, and thus $\left(\Delta z_{i+1} / \Delta z_{i}\right)=\left(\Delta_{X}{ }_{i+1} / \Delta X_{i}\right)^{2-D}$, from which the self-affine fractal dimension for the contour of the Cantor structure is derived as:

$$
D=1+\frac{\ln 2}{\ln 2 a}-\frac{\ln b}{\ln 2 a}=1+D_{c}-\frac{\ln b}{\ln 2 a} \quad \text { for }(1<D<2),
$$

where $D_{c}$ is the fractal dimension of the Cantor set $\left(0<D_{c}<1\right)$. Equation (2.3) will be used in the next section in the development of the approach-force model.

\section{The Continuous Elastic Model}

Qualitatively, two size scales are manifested in the contact problem [8]:

(1) the bulk scale, for which the elastic compression would be calculated by the Hertz theory and its limitations,

(2) the roughness scale, where the asperities act like a compliant layer on the surface, and so all the deformations are limited in a surface layer which represents all the asperities; $b h_{0}$ in Figure 2, and their deformation is assumed to be linear elastic [45].

In this paper, the approach of the punch of Cantor structure surface and length $L_{0}$ will be considered. It is to be noted that the obtained relation may be applied for all problems with surfaces having the same fractal dimension. The contact between two rough surfaces can be modeled as the contact of an effective surface with a rigid flat surface [10]. Hence, a solution for the deformation of an equivalent surface generated using the Cantor structure can be modified for the problem at hand. The bodies treated in this work will be assumed to be isotropic and homogeneous, and obey linear force-displacement laws. The yield strength $\sigma_{y}$, the modulus of elasticity $E$, and coefficient of thermal expansion $\alpha$, are all assumed to be independent of temperature.

Furthermore, it is assumed, with reference to Figure 2, that there exists a series of onedimensional elastic bars, distributed in a way such that the distance from the initiator step $E_{0}$ to the generated step $E_{3}$ is indicated by $h_{0}$, from $E_{1}$ to $E_{3}$ is indicated by $h_{1}$, from $E_{2}$ to $E_{3}$ is indicated by $h_{2}$, and so forth, [14-16]. By letting $F_{3}$ be the force required to compress $E_{3}$ 
until $E_{2}, F_{2}$ be the force require to compress $E_{3}$ and $E_{2}$ until $E_{1}$, and $F_{1}$ be the force required to compress $E_{3}, E_{2}$, and $E_{1}$ until $E_{0}$, and assuming unit depth, one obtains the following discrete force-displacement relations:

$$
\begin{gathered}
F_{3}=h_{2} k_{3}, \\
F_{2}=\left(h_{1}-h_{2}\right) k_{2}+h_{1} k_{3}, \\
F_{1}=\left(h_{0}-h_{1}\right) k_{1}+\left(h_{0}-h_{2}\right) k_{2}+h_{0} k_{3},
\end{gathered}
$$

where $k_{i}=E L_{i} / b h_{0}$ is the stiffness of the $i$ th step, $E$ is the modulus of elasticity of the material used, $b h_{0}$ could be understood from Figure 2, and $h_{i}$ and $L_{i}$ can be calculated using (2.1) and (2.2), respectively.

It is to be noted that thermal forces and deflections may arise in heated body either because of a nonuniform temperature distribution, or external constraints, or as a combination of these causes. The problem is assumed to be a steady state one with no internal heat source.

Next, by letting $\Delta F_{i+1}=F_{i}-F_{i+1}$, then from (3.1) one can conclude the general equation for any number of steps as follows:

$$
\Delta F_{i+1}=\frac{E L_{0}}{b}(b-1) b^{-i} a^{-i}
$$

In order to find a recursive relation as in (3.2) for the approach $u$, one lets $F_{i+1}$ be the limit force for protrusion of the $(i+1)$ th generation. It is assumed that when the limit load is reached, the punch approaches a distance $\Delta u_{i+1}$, equals to the difference between the heights protrusion of $i$ th and $(i+1)$ th generations. Consequently the second generation $E_{2}$ deflects a distance $u_{2}=h_{1}, E_{1}$ deflects a distance $u_{1}=h_{0}$, and $E_{0}$ deflects a distance $u_{0}=b h_{0}$, so $\Delta u_{1}=u_{0}-u_{1}=h_{0}(b-1)$, and $\Delta u_{2}=h_{0} b^{-1}(b-1)$. Accordingly:

$$
\Delta u_{i+1}=h_{0}(b-1) b^{-i}
$$

The above-mentioned assumptions are sufficient to determine the dependence of the limit load $F$ on the approach $u$. The effects of the remote load and the bulk temperature will be first studied separately and then superimposed. Using the fact that, when the limit load increases from $F_{i+1}$ to $F_{i}$, the punch is approached by an amount $\Delta u_{i+1}$, and by utilizing (3.2) and (3.3), the remote load effect is given by the discrete force displacement relation:

$$
\frac{\Delta F_{i+1}}{\Delta u_{i+1}}=\frac{E L_{0}}{b h_{0}} a^{-i}
$$

As $i \rightarrow \infty$, (3.4) yields the following asymptotic behavior for the strain $\varepsilon$ :

$$
\varepsilon=\left(\frac{b_{\chi}}{E} \frac{F}{L_{0}}\right)^{1 /(x+1)}
$$


where the strain $\varepsilon$ could be defined as $u / h_{0}$ and $\chi=\ln a / \ln b$ [14-16]. It is to be noted that, the effect of the applied external load should not exceed a limiting yield load $F_{y}$, where $F_{y}=\sigma_{y} L_{0}$.

Since the interest of this work is to consider the viscoelastic behavior, the principle of correspondence [44] will be used in the next section to obtain a viscoelastic model corresponding to the elastic model presented in (3.5).

\section{Effect of Temperature}

Temperature has a dramatic influence on rates of viscoelastic response, and in practical work it is often necessary to adjust a viscoelastic analysis for varying temperature. This strong dependence of temperature can also be useful in experimental characterization, for example, if a viscoelastic transition occurs too quickly at room temperature, for easy measurement, the experimenter can lower the temperature to slow things down and vice versa.

In some viscoelastic materials, the relation between time and temperature can be described by correspondingly simple models. Such materials are termed "thermorheologically simple" [46]. For such simple materials, the effect of lowering the temperature is simply to shift the viscoelastic response (plotted against log-time) to the right without change in shape. This is equivalent to increasing the relaxation time $\tau$, without changing the relaxation modulus.

A time-temperature shift factor $\left(a_{T}\right)$ can be defined as the horizontal shift that must be applied to a response curve, measured at an arbitrary temperature $T$ in order to move it to the curve measured at some reference temperature $T_{\text {ref. }}$.

If the creep time obeys an Arrhenius relation, the shift factor can be shown to be [47]:

$$
\log a_{T}=\frac{Q}{2.303 R}\left(\frac{1}{T}-\frac{1}{T_{0}}\right)
$$

where $Q$ is the activation energy $(\mathrm{J} / \mathrm{mol}), R$ is the gas constant $(\mathrm{J} / \mathrm{mol} \cdot \mathrm{K})$, and $T$ is the temperature $(\mathrm{K})$.

\section{Arrhenius Relation}

The creep properties of materials are usually described by reference to the dependence of the creep $(\varepsilon)$ on the applied stress, time and temperature, which may be written as:

$$
\varepsilon=f(\sigma, t, T) .
$$

One way to simplify this function is to make it to be separable into three functions of stress, time and temperature as follows [38]:

$$
\varepsilon=f_{1}(\sigma) f_{2}(t) f_{3}(T)
$$

Temperature has a significant effect on the creep of materials. In some steels, it is found that the temperature has a pronounced effect than the strain rate [48]. 


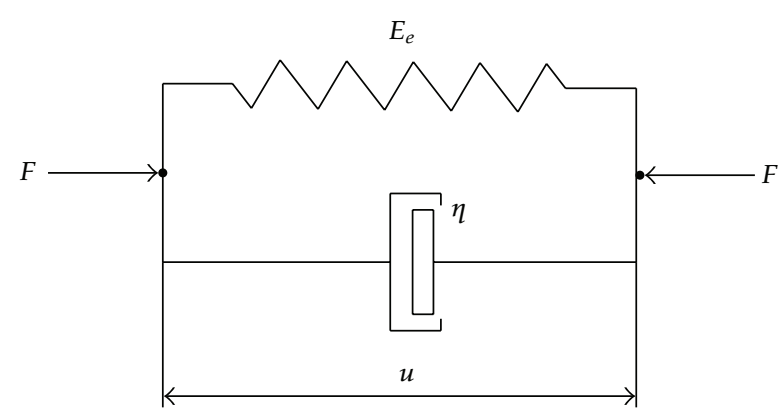

Figure 3: The linear Kelvin-Voigt model, where $\eta$ is the Newtonian viscosity, $E_{e}$ is the elastic modulus, $F$ is the applied load and $u$ indicates the points to be displaced.

Thermal forces and deflections may arise in a heated body either because of a nonuniform temperature distribution, or external constraints. The problem is assumed to be a steady state one with no internal heat. Arrhenius relation is a simple, but remarkably accurate, formula for the temperature dependence, where according to Arrhenius law, the temperature dependence is given as [49]:

$$
f_{3}(T)=B \exp \left(\frac{Q}{R T}\right)
$$

where $B$ is a constant, $Q$ is the activation energy and $R$ is the ideal gas constant. The functions $f_{1}(\sigma)$ and $f_{2}(t)$ will be included in (6.4) in Section 6.

It is clear that at the reference temperature, $T_{0}$, the temperature function $f_{3}(T)$ is equal to unity, and the creep will be a function of the stress and time, as it was shown in (5.1). From (5.3) at the reference temperature $T_{0}$, it can be easily shown that the value of the constant $B$ will be:

$$
B=\exp \left(\frac{-Q}{R T_{0}}\right)
$$

\section{Elastic Viscoelastic Correspondence}

The simplest approach to this problem consists of replacing the elastic constant in the elastic solution by the corresponding integral or differential operators from the viscoelastic stressstrain relations [44]. This approach can be applied to the contact problem provided that the loading program is such that the contact area is increasing throughout [38].

A Kelvin-Voigt linear model is employed to describe the viscoelastic behavior of the compliant layer. Such a model is an arrangement of spring and dashpot in parallel, as shown in Figure 3, in which $\eta$ and $E_{e}$ are the Newtonian viscosity and the elastic modulus, respectively. The time-dependent force-displacement relation could be written in the operator form using the linear differential time operator $\partial_{t} \equiv \partial / \partial t$ as shown in (6.1) [50]:

$$
\varepsilon=\frac{\sigma}{E_{e}+\eta \partial_{t}} .
$$


It is clear that simple the constant of proportionality between stress and strain does no longer exist. The viscoelastic operator corresponding to the modulus $E$ in (3.5) could be written as [50]:

$$
\frac{1}{E} \longrightarrow \frac{1}{E_{e}} \frac{1}{1+\tau \partial_{t}}
$$

where $\left(\tau \equiv \eta / E_{e}\right)$ is a characteristics parameter with units of time called the retardation time.

Creep test is a widely used standard test, wherein a force $P_{0}$ is suddenly applied at time $t=0$ on the viscoelastic model and then maintained constant thereafter, while measuring the approach as a function of time. The applied force can be expressed as a function of time with the aid of the unit step function $[U(t)]$. Thus

$$
F=P_{0}[U(t)] .
$$

By substituting (6.1)-(6.3) in (3.5) one obtains

$$
f_{1}(\sigma) f_{2}(t)=\left(\frac{u(t)}{h_{0}}\right)_{1}=\left(\frac{b_{\chi}}{\tau E_{e}} \frac{P_{0}}{L_{0}}\right)^{1 /(x+1)} \frac{t^{1 /(1+\chi)}}{\Gamma((2+x) /(1+\chi))}{ }_{1} F_{1}\left[\frac{1}{1+\chi} ; \frac{2+\chi}{1+\chi} ;-\frac{t}{\tau}\right]
$$

Tables of Laplace transforms [51] were utilized to obtain (6.4), where $\Gamma$ represent the gamma function, and ${ }_{1} F_{1}[c ; d ; x]$ is the Kummer's confluent hypergeometric function which could be expressed as [52]

$$
{ }_{1} F_{1}[c ; d ; x]=1+\frac{c}{d} x+\frac{c(c+1) x^{2}}{d(d+1) 2 !}+\frac{c(c+1)(c+2) x^{3}}{d(d+1)(d+2) 3 !}+\cdots
$$

or

$$
{ }_{1} F_{1}[c ; d ; x]=\sum_{n=0}^{\infty} \frac{(c)_{n}}{(d)_{n}} \frac{x^{n}}{n !} .
$$

By substituting the value of the constant $B$ from (5.4) into (5.3), and substituting (3.5) and (5.3) into (5.2), the creep stain as a function of stress, time, and temperature, is obtained as follows:

$$
\begin{aligned}
\varepsilon= & f_{1}(\sigma) f_{2}(t) f_{3}(T)=\frac{u(t)}{h_{0}}=\left(\frac{b_{\chi}}{\tau E_{e}} \frac{P_{0}}{L_{0}}\right)^{1 /(x+1)} \frac{t^{1 /(1+\chi)}}{\Gamma((2+\chi) /(1+\chi))}{ }_{1} F_{1}\left[\frac{1}{1+\chi} ; \frac{2+\chi}{1+\chi} ;-\frac{t}{\tau}\right] \\
& \times \exp \left(\frac{Q}{R}\left(\frac{1}{T}-\frac{1}{T_{0}}\right)\right),
\end{aligned}
$$

where $u(t) / h_{0}$ is the approach, $P_{0} / L_{0}$ is the applied stress per unit depth, $T$ is the bulk temperature, $T_{0}$ is the reference temperature, $\tau \equiv \eta / E_{e}$ is the retardation time, $\eta$ is the 


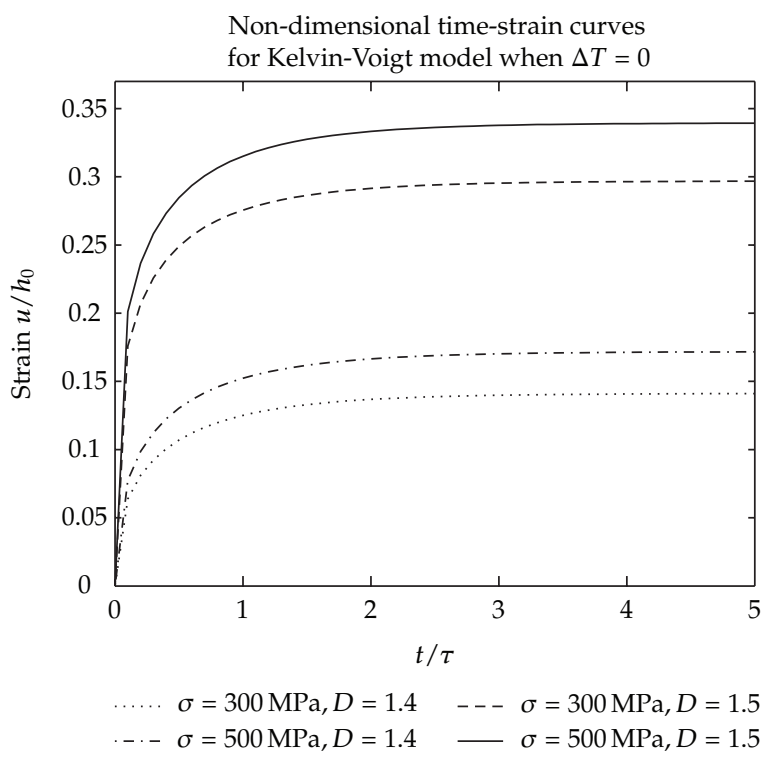

Figure 4: Non-dimensional time-strain curves for Kelvin-Voigt model when $\Delta T=0$, with $D=1.5$ and $D=1.4$, and two applied Stresses $\sigma_{1}=300 \mathrm{MPa}$ and $\sigma_{2}=500 \mathrm{MPa}$.

Newtonian viscosity, $E_{e}$ is the elastic modulus, $Q$ is the activation energy, and $R$ is the ideal gas constant. The effect of the fractal dimension $D$ appears through the constant $x$ which combines the two scaling parameters $\{a, b\}$, that is, $x=\ln a / \ln b$ (cf. (2.3)). The results obtained using this analytical model are presented and discussed in the next section.

\section{Results and Discussion}

A new continuous model for the creep contact of a thermovisco-elastic punch has been presented in (6.7). The model presents an approximate closed form solution for the approach $u(t) / h_{0}$ of the fractal surface as a function of the applied load $P_{0} / L_{0}$. In order to use (6.7), values for the system parameters $E_{e}, \eta, a$ and $b$ are needed. The constant parameters $a$ and $b$, in this equation, characterize the Cantor structure of the rough surface and are related through the fractal dimension $D$ of the rough surface. For the Kelvin-Voigt model to be capable of describing the experimental results of various viscoelastic materials, the viscous coefficients, $\eta(\mathrm{GPa} \cdot \mathrm{sec})$, and the elastic modulus $E_{e}(\mathrm{GPa})$, should be selected properly. In this study the modulus $E_{e}$ is selected to be $130 \mathrm{GPa}$ and the retardation time, $\tau \equiv \eta / E_{e}$, is selected to be of the order " 1 " [53]. The value of $n$ is taken large enough for the result to converge to an accepted accuracy; it is assumed that $n=550$.

The Cantor structure, shown in Figure 2, is built from the middle-third Cantor set where the parameter $a=1.5$ is held fixed, giving a Cantor set dimension $D_{c}=0.63093$ [18]. Two different values of the parameter $b(b=1.155$ and $b=1.29)$ yield two different dimensions of the Cantor structure; $D=1.5$ and $D=1.4$, respectively, which are used to verify the proposed analytical model. It was pointed out [11] that only for $b \leq 2$ the profile of the surface of the contacting body is fractal. 


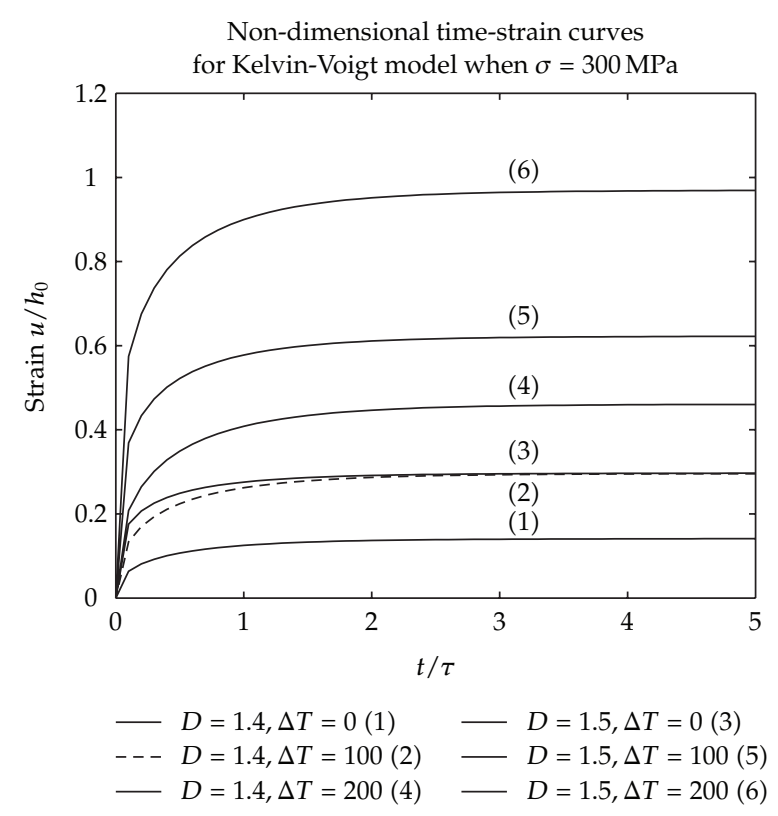

Figure 5: Non-dimensional time-strain curves for Kelvin-Voigt model when for three temperature differences $\Delta T=0,100$ and 200, with $D=1.5$ and $D=1.4$, and constant applied stress $\sigma=300 \mathrm{MPa}$.

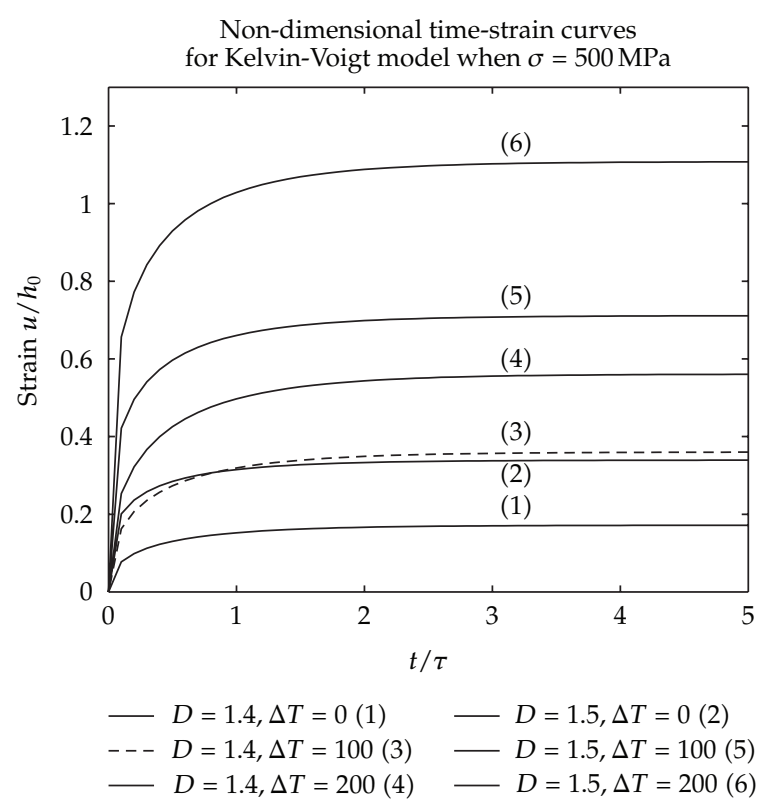

Figure 6: Non-dimensional time-strain curves for Kelvin-Voigt model when for three temperature differences $\Delta T=0,100$ and 200, with dimensions $D=1.5$ and $D=1.4$, and constant applied stress $\sigma=500 \mathrm{MPa}$. 


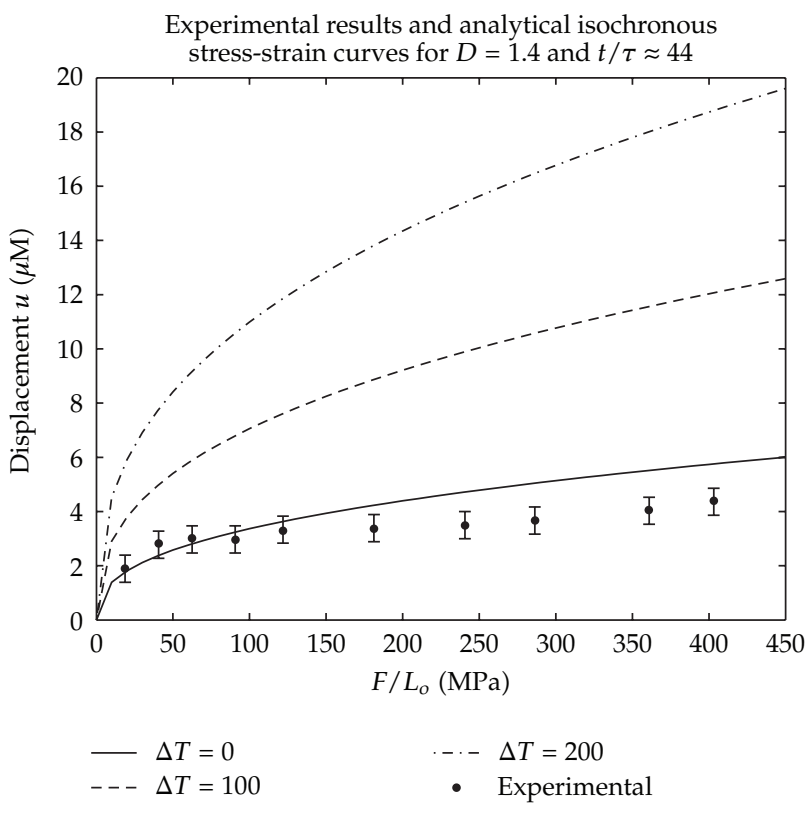

Figure 7: Experimental results and analytical isochronous Stress-Strain curves for constant $t / \tau \approx 44$ for three temperature differences $\Delta T=0, \Delta T=100$ and $\Delta T=200$, with $D=1.4$.

In order to examine the validity of the presented model, results obtained using this model for selected values of the system parameters were compared with those obtained experimentally by the results in $[12,54]$. These results displayed the approach-force relation between a flat rough surface and an ideally smooth and rigid counter surface. The specimens used in these experiments were made of carbon steel ( 0.45 percent carbon). Their surface roughness resulted from different finishing processes; face turning, grinding, and beadblasting. The experiments were conducted using MATLAB for a wide range of the nominal load, up to $600 \mathrm{MPa}$. The error in the experimental measurements was determined to be approximately $\mp 0.5 \mu \mathrm{m}$ for the approach, and $\mp 5 \mathrm{MPa}$ for the load [54]. Furthermore, the fractal dimension, $D$, of a ground stainless steel surfaces is $D=1.5$ [12]. The value of $h_{0}$, which corresponds to twice rms height for the ground surface, is taken as $6.6 \mu \mathrm{m}$ [54], and the value $D=1.5$ [37] is used to calculate the value of the parameter $b$, using (2.3), for a fixed value of the parameter $a$.

Figure 4 shows a set of numerical creep data obtained by applying the model which was presented in (6.7). In Figure 4 , the strain $u / h_{0}$ is plotted versus the nondimensional time record $t / \tau$ for $\Delta T=0$, with two different fractal dimensions $D=1.5$ and $D=1.4$, and two applied stresses $\sigma_{1}=300 \mathrm{MPa}$ and $\sigma_{2}=500 \mathrm{MPa}$. As might be expected, higher strain rates occur for higher temperatures for a constant stress.

Figure 5 shows the strain $u / h_{0}$ versus the nondimensional time record $t / \tau$. It shows, also, a set of numerical creep data obtained applying (6.7) for three temperature differences $\Delta T=0, \Delta T=100$ and $\Delta T=200$ with two different fractal dimensions $D=1.5$ and $D=1.4$, and constant applied stress $\sigma_{1}=300 \mathrm{MPa}$. The behavior is also similar to the known typical creep curves; it indicates that higher strain rates occur for higher temperatures.

Figure 6 is similar to Figure 5 in all of its aspects except for the applied stress, where in this case $\sigma=500 \mathrm{MPa}$. 


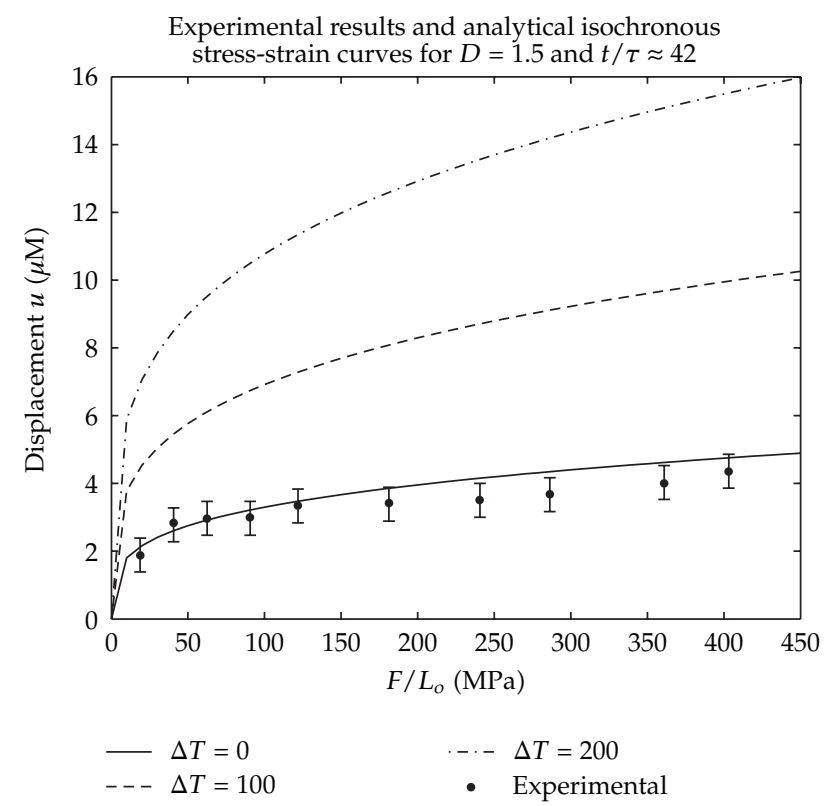

Figure 8: Experimental results and analytical isochronous Stress-Strain curves for constant $t / \tau \approx 42$ for three temperature differences $\Delta T=0, \Delta T=100$ and $\Delta T=200$, with $D=1.5$.

Figures 4-6 show that higher strain rates result for higher fractal dimensions, which could be explained from the definition of the fractal dimension itself. Lower fractal dimensions means less roughness and consequently the bulk material dominates, while higher fractal dimensions means excessive roughness and consequently the asperities deformation dominate.

Figure 7 presents the isochronous stress-strain creep curves accompanied by experimental results available in the literature [54] for nondimensional time durations $t / \tau \approx 44$ (which is held constant), fractal dimension $D=1.4$, and for three temperature differences $\Delta T=0, \Delta T=100$ and $\Delta T=200$.

Figure 8 also presents the isochronous stress-strain creep curves accompanied by experimental results available in the literature [54] which are conducted at the room temperature. The analytical results are shown for the nondimensional time durations $t / \tau \approx 42$ (which is held constant), fractal dimension $D=1.5$, and for temperature differences $\Delta T=0$, $\Delta T=100$ and $\Delta T=200$.

Figures 7 and 8 show good agreement between the presented proposed model and the experimental data. For $D=1.4$ the nondimensional time duration, $t / \tau$, required to get an agreement between the experimental results and the isochronous curves is about 44.2 , while it is about 44 for the fractal dimension $D=1.5$.

It is clear that the relatively longer duration of agreement occurs for lower fractal dimensions which could be attributed to the same reason mentioned above, that is, lower fractal dimensions means less roughness and consequently the bulk material dominates, while higher fractal dimensions means excessive roughness and consequently the asperities deformation dominate. The mathematical model also shows instability when $t / \tau$ exceeds 45 . 


\section{Conclusions and Future Work}

As well known, creep analysis is a nonlinear time-dependent phenomenon. The model which is modified in this work presents a solution to the thermal creep-contact of rough surfaces as a hypergeometric time series. Fractal geometry, via Cantor set, is utilized to model roughness of the creeping contact surfaces. The results obtained by this model turn out not to be too far from reality, since tests, at room temperatures, on the actual contact area of ground metal surfaces show that they contain sets of parallel ragged-edged scratches of different depths.

Since the construction of the Cantor structure is periodic in its nature, it undergoes the same construction procedure at each hierarchical level producing contact areas that are all of the same size. Therefore, the presented analytical model provides an approximate, not exact, simulation of the approach of the viscoelastic rough surfaces, where the presented model shows a fairly good agreement with the available experimental results. As linearity is an inherent assumption, it is not expected from this model to be able to describe exactly the real material behavior; roughness and deformation. A nonlinear thermoviscoelastic stressstrain relation is required for the reproduction of real material behavior. It is also clear that the specific character of the fractal model has little effect on the asymptotic behavior of the process, and the fractal dimension $D$ which provides a measure of the rate at which a surface is changing is of most importance. The solution obtained in this work provides further insight into the effect that surface structure has on the deformation process, and it also provides indications of the effect that different surface forming processes may have on the subsequent surface deformation. Furthermore, in the averaged sense, the Cantor structure model appears to provide fairly reasonable results.

For future work, it is intended to extend the methodology which is used in this paper for the application to the contact-surfaces within electronic and electrical devices and circuits such as resistors, capacitors and inductors that arise in electronic manufacturing systems. Furthermore, it is intended to further investigate the applications of fractals in the emerging quantum computing domain.

\section{References}

[1] F. M. Borodich and D. A. Onishchenko, "Similarity and fractality in the modelling of roughness by a multilevel profile with hierarchical structure," International Journal of Solids and Structures, vol. 36, no. 17, pp. 2585-2612, 1999.

[2] J. A. Greenwood, "Problems with surface roughness," in Fundamentals of Friction: Macroscopic and Microscopic Processes, I. L. Singer and H. M. Pollock, Eds., pp. 57-76, Kluwer, Boston, Mass, USA, 1992.

[3] A. Majumdar and B. Bhushan, "Role of fractal geometry in roughness characterization and contact mechanics of surfaces," Journal of Tribology, vol. 112, no. 2, pp. 205-216, 1990.

[4] B. B. Mandelbrot, D. E. Passoja, and A. J. Paullay, "Fractal character of fracture surfaces of metals," Nature, vol. 308, no. 5961, pp. 721-722, 1984.

[5] M. Li and W. Zhao, "Representation of a stochastic traffic bound," to appear in IEEE Transactions on Parallel and Distributed Systems, IEEE Computer Society Digital Library, IEEE Computer Society, http:/ / doi.ieeecomputersociety. org/10.1109/TPDS.2009.162.

[6] M. Li and S. C. Lim, "Modeling network traffic using generalized Cauchy process," Physica A, vol. 387, no. 11, pp. 2584-2594, 2008.

[7] M. Li, "Generation of teletraffic of generalized Cauchy type," Physica Scripta, vol. 82, no. 2, Article ID 025007, 2010.

[8] K. L. Johnson, Contact Mechanics, Cambridge University Press, Cambridge, UK, 1985.

[9] J. A. Greenwood and J. B. P. Williamson, "Contact of nominally flat surfaces," Proceedings of the Royal Society of London. Series A, vol. 295, no. 1442, pp. 300-319, 1966. 
[10] A. Majumdar and B. Bhushan, "Fractal model of elastic-plastic contact between rough surfaces," Journal of Tribology, vol. 113, pp. 1-11, 1991.

[11] F. M. Borodich and A. B. Mosolov, "Fractal roughness in contact problems," Journal of Applied Mathematics and Mechanics, vol. 56, no. 5, pp. 786-795, 1992.

[12] T. L. Warren and D. Krajcinovic, "Fractal models of elastic-perfectly plastic contact of rough surfaces based on the Cantor set," International Journal of Solids and Structures, vol. 32, no. 19, pp. 2907-2922, 1995.

[13] T. L. Warren, A. Majumdar, and D. Krajcinovic, "A fractal model for the rigid-perfectly plastic contact of rough surfaces," Journal of Applied Mechanics, vol. 63, no. 1, pp. 47-54, 1996.

[14] O. Abuzeid, "Linear viscoelastic creep model for the contact of nominal flat surfaces based on fractal geometry: Maxwell type medium," Dirasat-Engineering Sciences, The University of Jordan, vol. 30, no. 1, pp. 22-36, 2003.

[15] O. M. Abuzeid and P. Eberhard, "Linear viscoelastic creep model for the contact of nominal flat surfaces based on fractal geometry: standard linear solid (SLS) material," Journal of Tribology, vol. 129, no. 3, pp. 461-466, 2007.

[16] O. M. Abuzeid and T. A. Alabed, "Mathematical modeling of the thermal relaxation of nominally flat surfaces in contact using fractal geometry: Maxwell type medium," Tribology International, vol. 42, no. 2, pp. 206-212, 2009.

[17] F. Borodich, "Fractals and surface roughness in EHL," in IUTAM Symposium on Elastohydrodynamics and Micro-Elastohydrodynamics, R. Snidle and H. Evans, Eds., vol. 134 of Solid Mechanics and Its Applications, pp. 397-408, Springer, Dordrecht, The Netherlands, 2006.

[18] B. B. Mandelbrot, The Fractal Geometry of Nature, W. H. Freeman, San Francisco, Calif, USA, 1982.

[19] R. D. Mauldin and S. C. Williams, "On the Hausdorff dimension of some graphs," Transactions of the American Mathematical Society, vol. 298, no. 2, pp. 793-803, 1986.

[20] D. Wojcik, I. Białynicki-Birula, and K. Zyczkowski, "Time evolution of quantum fractals," Physical Review Letters, vol. 85, no. 24, pp. 5022-5025, 2000.

[21] A. N. Al-Rabadi, Reversible Logic Synthesis: From Fundamentals to Quantum Computing, Springer, Berlin, Germany, 2004.

[22] C. Cattani and A. Kudreyko, "Application of periodized harmonic wavelets towards solution of eigenvalue problems for integral equations," Mathematical Problems in Engineering, vol. 2010, Article ID 570136, 8 pages, 2010.

[23] E. G. Bakhoum and C. Toma, "Dynamical aspects of macroscopic and quantum transitions due to coherence function and time series events," Mathematical Problems in Engineering, vol. 2010, Article ID 428903, 13 pages, 2010.

[24] G. Toma, "Specific differential equations for generating pulse sequences," Mathematical Problems in Engineering, vol. 2010, Article ID 324818, 11 pages, 2010.

[25] G. Mattioli, M. Scalia, and C. Cattani, "Analysis of large amplitude pulses in short time intervals: application to neuron interactions," Mathematical Problems in Engineering, vol. 2010, Article ID 895785, 15 pages, 2010.

[26] S. Y. Chen, Y. F. Li, and J. Zhang, "Vision processing for realtime 3-D data acquisition based on coded structured light," IEEE Transactions on Image Processing, vol. 17, no. 2, pp. 167-176, 2008.

[27] S. Y. Chen, Y. F. Li, Q. Guan, and G. Xiao, “Real-time three-dimensional surface measurement by color encoded light projection," Applied Physics Letters, vol. 89, no. 11, Article ID 111108, 2006.

[28] K. Falconer, Fractal Geometry: Mathematical Foundations and Applications, John Wiley \& Sons, Chichester, UK, 1990.

[29] P. S. Modenov and A. S. Parkhomenko, Geometric Transformations. Vol. 1: Euclidean and Affine Transformations, Academic Press, New York, NY, USA, 1965.

[30] R. F. Voss, "Random fractal forgeries," in Fundamental Algorithms in Computer Graphics, R. A. Earnshaw, Ed., pp. 805-835, Springer, Berlin, Germany, 1985.

[31] A. Majumdar and C. L. Tien, "Fractal characterization and simulation of rough surfaces," Wear, vol. 136, no. 2, pp. 313-327, 1990.

[32] J. Lopez, G. Hansali, H. Zahouani, J. C. Le Bosse, and T. Mathia, “3D fractal-based characterisation for engineered surface topography," International Journal of Machine Tools and Manufacture, vol. 35, no. 2, pp. 211-217, 1995.

[33] M. Li, "Fractal time series-a tutorial review," Mathematical Problems in Engineering, vol. 2010, Article ID 157264, 26 pages, 2010.

[34] M. Li and J.-Y. Li, "On the predictability of long-range dependent series," Mathematical Problems in Engineering, vol. 2010, Article ID 397454, 9 pages, 2010. 
[35] R. S. Sayles and T. R. Thomas, "Surface topography as a nonstationary random process," Nature, vol. 271, no. 5644, pp. 431-434, 1978.

[36] S. R. Brown, "Simple mathematical model of a rough fracture," Journal of Geophysical Research, vol. 100, no. 4, pp. 5941-5952, 1995.

[37] M. V. Berry and Z. V. Lewis, "On the Weierstrass-Mandelbrot fractal function," Proceedings of the Royal Society of London. Series A, vol. 370, no. 1743, pp. 459-484, 1980.

[38] E. H. Lee and J. R. M. Radok, "The contact problem for viscoelastic bodies," Journal of Applied Mechanics, vol. 27, pp. 438-444, 1960.

[39] T. C. T. Ting, "The contact stress between a rigid indenter and a viscoelastic half-space," Journal of Applied Mechanics, vol. 33, pp. 845-854, 1966.

[40] T. C. T. Ting, "Contact problems in the linear theory of viscoelasticity," Journal of Applied Mechanics, vol. 35, pp. 248-254, 1968.

[41] G. R. Nghieh, H. Rahnejat, and Z. M. Jin, "Contact mechanics of viscoelastic layered surface," in Contact Mechanics III, M. H. Aliabadi and A. Samartin, Eds., pp. 59-68, Computational Mechanics Publications, Boston, Mass, USA, 1997.

[42] K. J. Wahl, S. V. Stepnowski, and W. N. Unertl, "Viscoelastic effects in nanometer-scale contacts under shear," Tribology Letters, vol. 5, no. 1, pp. 103-107, 1998.

[43] D. J. Whitehouse and J. F. Archard, "The properties of random surfaces of significance in their contact," Proceedings of the Royal Society of London. Series A, vol. 316, pp. 97-121, 1970.

[44] J. R. M. Radok, "Visco-elastic stress analysis," Quarterly of Applied Mathematics, vol. 15, pp. 198-202, 1957.

[45] P. E. D'yachenko, N. N. Tolkacheva, G. A. Andreev, and T. M. Karpova, The Actual Contact Area between Touching Surfaces, Consultant Bureau, New York, NY, USA, 1964.

[46] N. J. Distefano and K. S. Pister, "On the identification problem for thermorheologically simple materials," Acta Mechanica, vol. 13, no. 3-4, pp. 179-190, 1972.

[47] T. Junisbekov, V. Kestelman, and N. Malinin, Stress Relaxation in Viscoelastic Materials, Science Publishers, Enfield, NH, USA, 2nd edition, 2003.

[48] W.-S. Lee and C.-Y. Liu, "The effects of temperature and strain rate on the dynamic flow behaviour of different steels," Materials Science and Engineering A, vol. 426, no. 1-2, pp. 101-113, 2006.

[49] J. Boyle and J. Spencer, Stress Analysis for Creep, Butterworths-Heinemann, London, UK, 1st edition, 1983.

[50] I. H. Shames and F. A. Cozzarelli, Elastic and Inelastic Stress Analysis, Prentice-Hall International, Englewood Cliffs, NJ, USA, 1992.

[51] G. E. Roberts and H. Kaufman, Table of Laplace Transforms, W. B. Saunders, Philadelphia, Pa, USA, 1966.

[52] L. J. Slater, Confluent Hypergeometric Functions, Cambridge University Press, New York, NY, USA, 1960.

[53] W. Nowacki, Thermoelasticity, Pergamon Press, Oxford, UK, 2nd edition, 1986.

[54] Z. Handzel-Powierza, T. Klimczak, and A. Polijaniuk, "On the experimental verification of the Greenwood-Williamson model for the contact of rough surfaces," Wear, vol. 154, no. 1, pp. 115-124, 1992. 


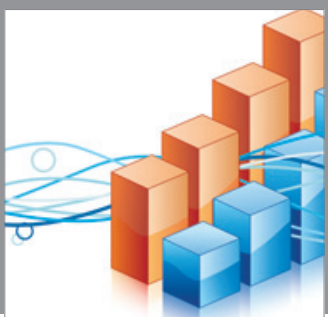

Advances in

Operations Research

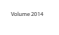

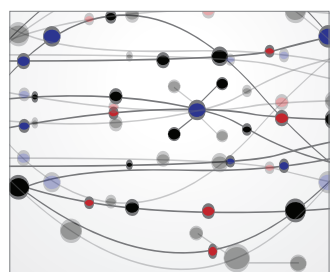

\section{The Scientific} World Journal
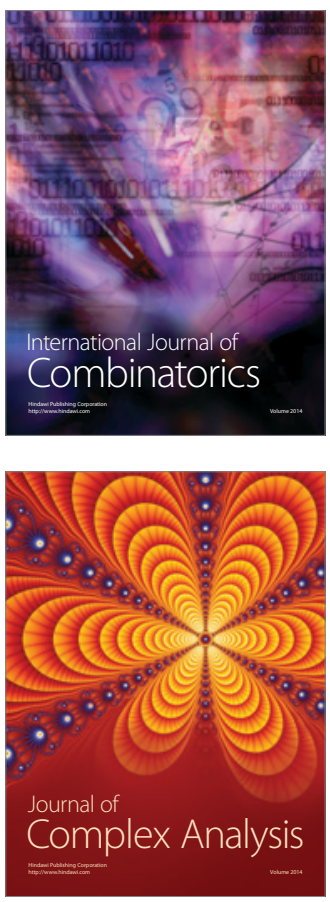

International Journal of

Mathematics and

Mathematical

Sciences
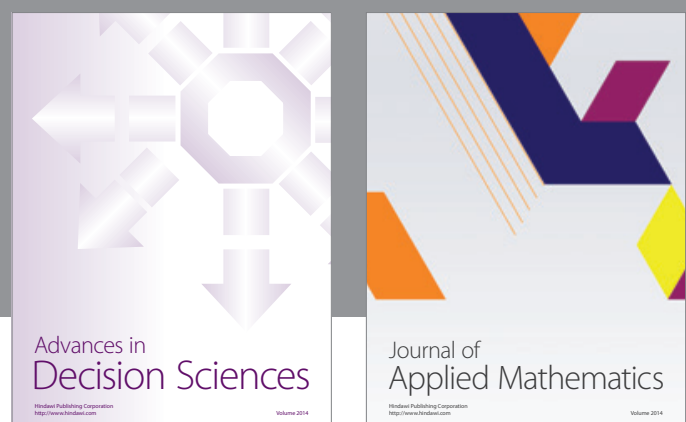

Journal of

Applied Mathematics
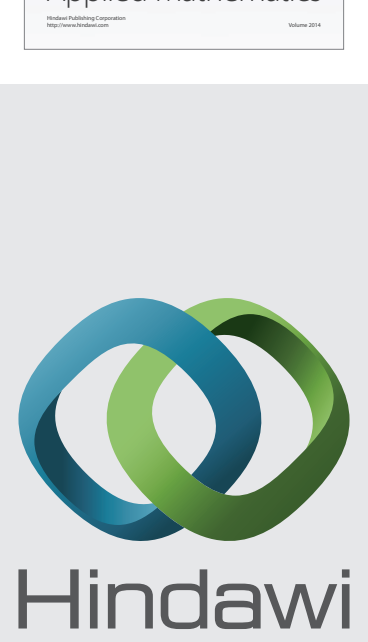

Submit your manuscripts at http://www.hindawi.com
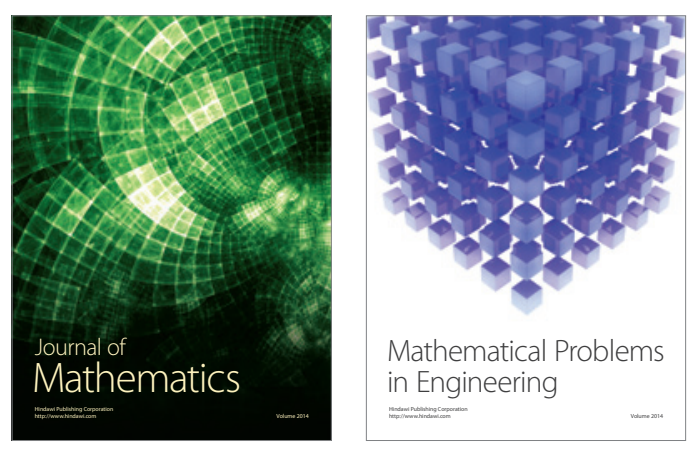

Mathematical Problems in Engineering
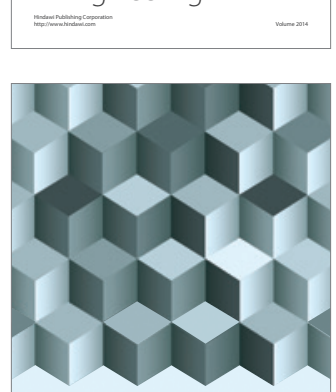

Journal of

Function Spaces
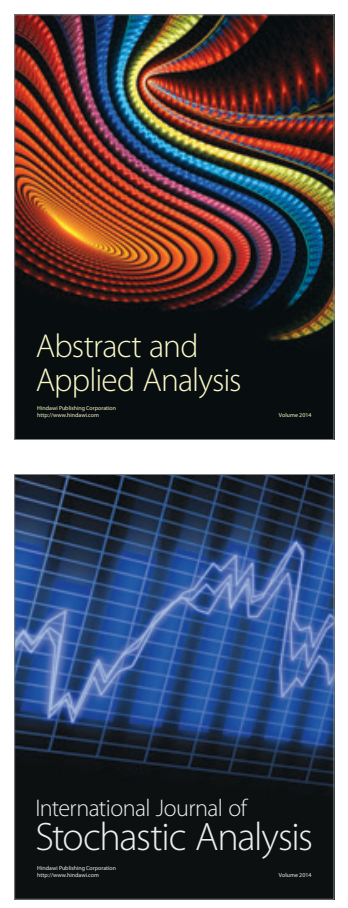

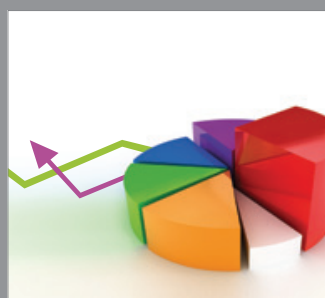

ournal of

Probability and Statistics

Promensencen
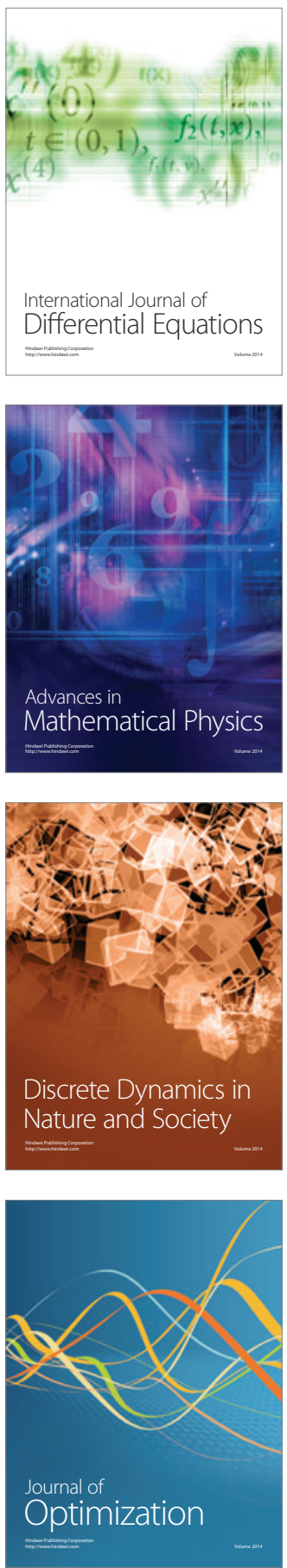